\title{
A NOTE ON A THEOREM BY H. D. BRUNK ${ }^{1}$
}

\section{RICHARD DYKSTRA ${ }^{2}$}

Throughout the paper, the notation will be consistent with that used by H. D. Brunk in [1]. That is $(\Omega, \delta, \mu)$ is a complete measure space, and $L_{2}$ denotes the set of square integrable functions corresponding to it. We shall call $\&$, a collection of sets in $\mathcal{S}$, a sub- $\sigma$-lattice if it is closed under countable unions and intersections, and contains the null set $\varnothing$, and $\Omega$. A function $X$ is $\mathscr{L}$-measurable if $[X>a] \in \mathscr{L}$ for all real $a . L_{2}(\mathscr{L})$ denotes the set of $\mathscr{L}$-measurable functions which are also in $L_{2}$. A family $C$ of measurable functions is called a convex cone if $k \geqq 0, X \in C, Y \in C \Rightarrow k X \in C, X+Y \in C$. A collection of functions is a lattice if the pointwise supremum and infinum of any two functions in the collection is in the collection. If $M$ is a collection of functions, $-M=\{-X: X \in M\}$. Similarly, $\mathscr{L}^{c}=\left\{A: A^{c} \in \mathfrak{L}\right\} . I_{A}$ or $I(A)$ will be the indicator function of the set $A$.

In [1], H. D. Brunk stated the following theorem.

THEOREM. $M$, a subset of $L_{2}$ is $L_{2}(\mathfrak{L})$ for some $\sigma$-lattice $\mathfrak{L}$ containing $\Phi$ and $\Omega$ if and only if

(1) $M$ is a lattice closed in $L_{2}$;

(2) a real, $X \in M, A=[X>a], \mu(A)<\infty$ implies $I(A) \in M$; a real, $X \in M, A=[X \geqq a], \mu\left(A^{c}\right)<\infty$ implies $-I\left(A^{c}\right) \in M$;

(3) $M$ is a convex cone.

However the theorem is not quite true as stated, for if $M$ is the set of nonnegative functions which are also square-integrable with respect to the measure space of the reals, Borel sets, and Lebesque measure, then $M$ satisfies the conditions of the theorem, yet $M$ is not $L_{2}(\mathfrak{L})$ for any $\sigma$-lattice $\mathscr{L}$.

However, if we slightly change (2), we can drop the requirement that $M$ be a lattice to obtain the following theorem.

THEOREM. $M$, a nonempty subset of $L_{2}$, is $L_{2}(\mathfrak{L})$ for some $\sigma$-lattice $\mathfrak{L}$ containing $\Phi$ and $\Omega$ if and only if

(1) $M$ is a convex cone closed in $L_{2}$;

(2) $a \geqq 0, \quad X \in M(-M), \quad A=[X \geqq a], \quad \mu(A)<\infty$ implies $I(A)$ $\in M(-M)$.

Received by the editors August 16, 1968.

1 This investigation was supported in part by PHS Research Grant No. GM 14448-01 from the Division of General Medical Sciences.

2 Currently at the University of Missouri. 
Proof. (The proof incorporates many of the arguments given in Brunk's proof as well as the notation used in [1].) To show necessity, note that it is easy to verify that $L_{2}(\mathscr{L})$ is a convex cone since $[X+Y>a]=U_{r}\{[X>a-r] \cap[Y>r]\}$ where the union is taken over the set of rational numbers. To show that $L_{2}(\mathscr{L})$ is closed in $L_{2}$, assume that $f_{n} \stackrel{L_{2}}{\rightarrow} f$, where $f_{n} \in L_{2}(\mathfrak{L})$ for all $n$. Then there exists, a subsequence $f_{n_{j}}$ such that $f_{n_{j}}^{\text {a.e. }} \rightarrow f$. Now for each real number $a$,

$$
[f>a]=\bigcup_{m=1}^{\infty} \bigcup_{k=1}^{\infty} \bigcap_{j=k}^{\infty}\left[f_{n_{j}}>a+1 / m\right]
$$

which belongs to $\mathscr{L}$ since $\mathscr{L}$ is closed under countable unions and intersections. Thus $f \in L_{2}(\&)$, so that $L_{2}(\&)$ is closed in $L_{2}$. It is easy to verify that $L_{2}(\mathscr{L})$ also satisfies (2).

Let us now be concerned with showing the sufficiency. Observe first of all that $\mu([X>a])<\infty$, where $X \in M$ and $a \geqq 0$, implies that $I([X>a]) \in M$ since $I([X \geqq a+1 / n]) \in M$ for all $n$ by (2) and converges to $I([X>a])$ in $L_{2}$.

Now let $\mathcal{L}^{+}=\{[X>a] ; a \geqq 0, X \in M\}$ and

$$
\mathscr{L}^{-}=\{[X \geqq-a] ; \quad a \geqq 0, X \in M] .
$$

We will show that $\mathscr{L}^{+}$is closed under countable unions and countable intersections. Let $C$ be a countable union of sets in $\mathscr{L}^{+}$. Then since $[X>0]=\bigcup_{n=1}^{\infty}[X>1 / n]$, we may assume that $C=\bigcup_{i=1}^{\infty} C_{i}$ where $0<\mu\left(C_{i}\right)<\infty$ and $C_{i} \in \mathcal{L}^{+}$. Define $Y_{n}=\sum_{i=1}^{n} I\left(C_{i}\right)\left[2^{i} \mu\left(C_{i}\right) \vee 2^{i}\right]^{-1}$ where $2^{i} \mu\left(C_{i}\right) \bigvee 2_{i}$ denotes the supremum of $2^{i} \mu\left(C_{i}\right)$ and $2^{i}$. Then $Y_{n} \in M$ for all $n$ by (1). Since $\left\{Y_{n}\right\}$ is a monotone nondecreasing sequence of functions bounded above by $1, Y=\lim _{n \rightarrow \infty} Y_{n}$ is well defined. Yet

$$
\begin{aligned}
\lim _{n \rightarrow \infty} \int\left(Y-Y_{n}\right)^{2} d \mu & =\lim _{n \rightarrow \infty} \int\left(\sum_{i=n+1}^{\infty} I\left(C_{i}\right)\left[2^{i} \mu\left(C_{i}\right) \vee 2^{i}\right]^{-1}\right)^{2} d \mu \\
& \leqq \lim _{n \rightarrow \infty} \int \sum_{i=n+1}^{\infty} I\left(C_{i}\right)\left[2^{i} \mu\left(C_{i}\right) \vee 2^{i}\right]^{-1} d \mu \\
& =\lim _{n \rightarrow \infty} \sum_{i=n+1}^{\infty}\left[2^{i} \mu\left(C_{i}\right) \bigvee 2^{i}\right]^{-1} \int I\left(C_{i}\right) d \mu \\
& \leqq \lim _{n \rightarrow \infty} \sum_{i=n+1}^{\infty}\left[2^{i} \mu\left(C_{i}\right)\right]^{-1} \mu\left(C_{i}\right) \\
& =\lim _{n \rightarrow \infty} \sum_{i=n+1}^{\infty} 1 / 2^{i}=0
\end{aligned}
$$


since one can interchange the summation sign with the integral in the case of nonnegative functions. Thus $Y \in M$ by (1). But it is easily shown that $C=\bigcup_{i=1}^{\infty} C_{i}=[Y>0] \in \mathfrak{L}^{+}$, so that $\mathfrak{L}^{+}$is closed under countable unions.

Suppose now that $C$ is a countable intersection of sets in $\mathfrak{L}^{+}$. Then we may assume that $C=\bigcap_{i=1}^{\infty} C_{i}$ where $C_{i}=\left[X_{i}>0\right], X_{i} \in M$, since $[X>a]=[I([X>a])>0]$ where $a>0$. Let $A_{n}=\left[X_{1}>1 / n\right]$, so that $\bigcup_{n=1}^{\infty} A_{n}=C_{1}$. Then $\left[\left(I\left(A_{n}\right)+I\left(\left[X_{i}>1 / k\right]\right)>1\right]=A_{n} \cap\left[X_{i}>1 / k\right] \in \mathfrak{L}^{+}\right.$ for all positive integers $i, k$, and $n$. Thus $\bigcup_{k=1}^{\infty}\left\{A_{n} \cap\left[X_{i}>1 / k\right]\right\}$ $=A_{n} \cap\left\{\mathrm{U}_{k=1}^{\infty}\left[X_{i}>1 / k\right]\right\}=A_{n} \cap C_{i} \in \mathcal{L}^{+}$for all $i$ and $n$. By a previous argument, $Z_{n}=\lim _{m \rightarrow \infty} \sum_{i=2}^{m} I\left(A_{n} \cap C_{i}\right)\left[2^{i} \mu\left(A_{n} \cap C_{i}\right) \vee 2^{i}\right]^{-1} \in M$ for all $n$. Thus by (2), $I\left(\left[Z_{n} \geqq \sum_{i=2}^{\infty}\left[2_{\mu}^{i}\left(A_{n} \cap C_{i}\right) \vee 2^{i}\right]^{-1}\right]\right) \in M$ so that

$$
\begin{aligned}
{\left[I \left(\left[Z_{n} \geqq \sum_{i=2}^{\infty}\left[2^{i} \mu\left(A_{n} \cap C_{i}\right)\right.\right.\right.\right.} & \left.\left.\left.\left.\vee 2^{i}\right]^{-1}\right]\right)>0\right] \\
& =\bigcap_{i=2}^{\infty}\left[A_{n} \cap C_{i}\right]=A_{n} \cap\left(\bigcap_{i=2}^{\infty} C_{i}\right) \in \mathscr{L}^{+},
\end{aligned}
$$

so that $C=\bigcap_{i=1}^{\infty} C_{i}=\bigcup_{n=1}^{\infty}\left\{A_{n} \cap\left(\cap_{i=2}^{\infty} C_{i}\right)\right\} \in \mathcal{L}^{+}$.

Let us now show that $\mathfrak{L}^{-}$is also closed under countable unions and countable intersections. Clearly it will suffice to show that this is true for $\left(\mathscr{L}^{-}\right)^{c}$. But

$$
\begin{aligned}
\left(\mathscr{L}^{-}\right)^{c} & =\{[X<-a] ; a \geqq 0, X \in M\}=\{[-X>a] ; a \geqq 0, X \in M\} \\
& =\{[Y>a] ; a \geqq 0, Y \in-M\} .
\end{aligned}
$$

However, this is how $\mathfrak{L}^{+}$is defined with the exception that $M$ is replaced by $-M$. Clearly, $-M$ has precisely the properties that $M$ has, and hence $\left(\mathscr{L}^{-}\right)^{c}$ is closed under countable unions and countable intersections by an earlier part of the theorem.

If $A \in \mathfrak{L}^{+}$and $B \in \mathfrak{L}^{-}$where $\mu(A)<\infty$, and $\mu\left(B^{c}\right)<\infty$, then $Z=I(A)-I\left(B^{c}\right) \in M$, so that $A \cap B=[Z>0] \in \mathcal{L}^{+}$and $A \cup B$ $=[Z \geqq 0] \in \mathcal{L}^{-}$. In general, if $A \in \mathcal{L}^{+}$, and $B \in \mathcal{L}^{-}$, then there exists a sequence $\left\{A_{i}\right\} \in \mathfrak{L}^{+}$and a sequence $\left\{B_{i}\right\} \in \mathfrak{L}^{+}$such that $\mu\left(A_{i}\right)<\infty$, $\mu\left(B_{j}^{c}\right)<\infty$ for all positive integers $i$ and $j, A=\bigcup_{i=1}^{\infty} A_{i}$, and $B=\bigcap_{i=1}^{\infty} B_{j}$. Then $A \cap B=\bigcup_{i=1}^{\infty} \bigcap_{j=1}^{\infty}\left(A_{i} \cap B_{j}\right) \in \mathcal{L}^{+}$, and $A \cup B=\bigcap_{j=1}^{\infty} \bigcup_{i=1}^{\infty}\left(A_{i} \cup B_{j}\right)$ $\in \mathscr{L}^{-}$, so that $\mathscr{L}=\mathscr{L}^{+} \cup \mathfrak{L}^{-}$is a $\sigma$-lattice containing $\Phi$ and $\Omega$. It is easily shown that $M \subset L_{2}(\mathscr{L})$. The reverse inclusion can be shown by separating an arbitrary member $f$ of $L_{2}(\mathscr{L})$ into its positive and negative part, and approximating each part by the respective simple functions 


$$
f_{n}^{+}=\sum_{i=1}^{n 2^{n}} 1 / 2^{n} I\left[f^{+} \geqq i / 2^{n}\right]
$$

and

$$
-\overline{f_{n}}=-\sum_{i=1}^{n 2^{n}} 1 / 2^{n} I\left[-\bar{f} \leqq-i / 2^{n}\right],
$$

which belongs to $M$. Then using the fact that these simple functions converge in $L_{2}$ to the respective positive and negative parts of $f$, and the fact that $M$ is closed under addition, we have our desired result.

In [1], condition (2) can be replaced by the condition that $M$ contain all constant functions when $\mu(\Omega)<\infty$. The corresponding condition of the revised theorem cannot be weakened in this manner.

\section{REFERENCE}

1. H. D. Brunk, On an extension of the concept conditional expectation, Proc. Amer. Math. Soc. 14 (1963), 298-304. MR 26 \#5599. 